\title{
Case report series: management of complete ureteral avulsion with review of the relevant literature
}

\author{
İbrahim Ünal Sert and Arif Aydın* (10)
}

\begin{abstract}
Background: Endoscopic operations and ureteroscopy have become the first choice for ureteral calculi and ureterrelated operations. The ratios of the complications, which are mostly iatrogenic, range between 9 and $11 \%$. Total ureteral avulsion during URS is quite rare with a prevalence of $0-0.3 \%$. We present three total ureteral avulsions we experienced in our clinic during the last 2 years and their treatment.
\end{abstract}

Case presentation: During the last 2 years, we experienced three total ureteral avulsions: one of these occurred in our clinic and the other two occurred in an external center and were referred to us. In two cases, the omental flap was rotated after ureteral reimplantation and the ureter was completely wrapped inside the omental flap. In the third case, boari flap was formed from the bladder. Due to the presence of extrarenal wide renal pelvis, anastomosis was made with boari flap after a $y-v$ flap was rotated on the renal pelvis. D-J stents of the patients were removed at the end of 3 months. Although there was mild hydronephrosis, parenchymal thinning and a significant decrease in functions were not observed. Ureteral avulsion of the patients was successfully managed without the need for nephrectomy.

Conclusion: Ureteral avulsion management is an extremely difficult condition for both the surgeon and the patient. So, the most important thing is to prevent ureteral avulsion. Ureteroplasty and omental flap treatment are applicable methods with quite successful results for complete ureteral avulsions.

Keywords: Ureteroscopy, Complication, Ureteral avulsion, Ureteral avulsion treatment

\section{Background}

Due to its improvement over time, ureteroscopy (URS), started by Perez-Castro and Martinez Pineiro in 1980, has become the gold standard procedure for many clinical conditions affecting ureteral calculi, urethra and kidney [1, 2]. Ratios of mostly iatrogenic complications range between 9 and $11 \%$. Total ureteral avulsion during URS is quite rare with a prevalence of $0-0.3 \%$ [3]. This feared and severe complication is an extremely difficult condition for urologists and may necessitate nephrectomy while management requires experience $[4,5]$.

*Correspondence: aydinarif@gmail.com

Department of Urology, Medicine Faculty, NEÜ Meram, Konya, Turkey
Many treatments are recommended for this complication such as the following: kidney autotransplantation, ureterovesical anastomosis, ileal transposition, ureterocalicostomy and uretero-ureteral end-to-end anastomosis, boari flap, pyeloureterostomy, external wrapping of avulsified ureter with omentum and ureterovesical anastomosis, etc. [5-7]. Management of ureteral avulsion is a difficult condition, while end-to-end anastomoses constitute a good option for proximal ureteral avulsions, boari flap and psoas hitch methods are suggested if ureteral avulsion is in the middle one-third section. Reimplantation is suggested for distal ureteral injuries [4].

We present three total ureteral avulsions experienced in our clinic during the last 2 years and their treatments. 



Fig. 1 Ureteral re-implantation technique. a Degloving ureter, b D-j stent in ureter that anastomosis between bladder and kidney, c omental flap, d omental flap drawing

\subsection{Case presentations}

During the last 2 years, we experienced three total ureteral avulsions, one of which occurred in our clinic and the other two occurred in an external center and were referred to us. A total of three ureteral avulsion cases were intervened. Two of these patients were male and one was female.

\section{Case 1}

\subsection{Medical history}

A 66-year-old female patient referred to the clinic with right side pain and hematuria. An average degree of hydronephrosis in the right urinary system and an $8 \mathrm{~mm}$ stone in the mid-ureter were observed with computed tomography. Surgery for the patient was planned, and the right orifice was accessed with guided $9 \mathrm{~F}$ ureterorenoscope. An attempt was made to insert a $4.8 \mathrm{~F}$ D-J stent through the ureterorenoscope as the lower ureter was very narrow. Relief was felt in the ureterorenoscope during this period. When the ureterorenoscope was removed, the ureter was noticed at its end.

\subsection{Surgical technique}

A modified Gibson incision was performed for repair. The retroperitoneum was entered, and the ureter was reached and dissected. When bladder-ureter reanastomosis was about to be performed, it was noticed that the ureter was also separated from the ureteropelvic (UP) junction. Then, the avulsified ureter was kept in isotonic saline solution until anastomosis. Afterward, the UP junction was dissected and the avulsified ureter UP junction was anastomosed. D-J stent was inserted. Then, 

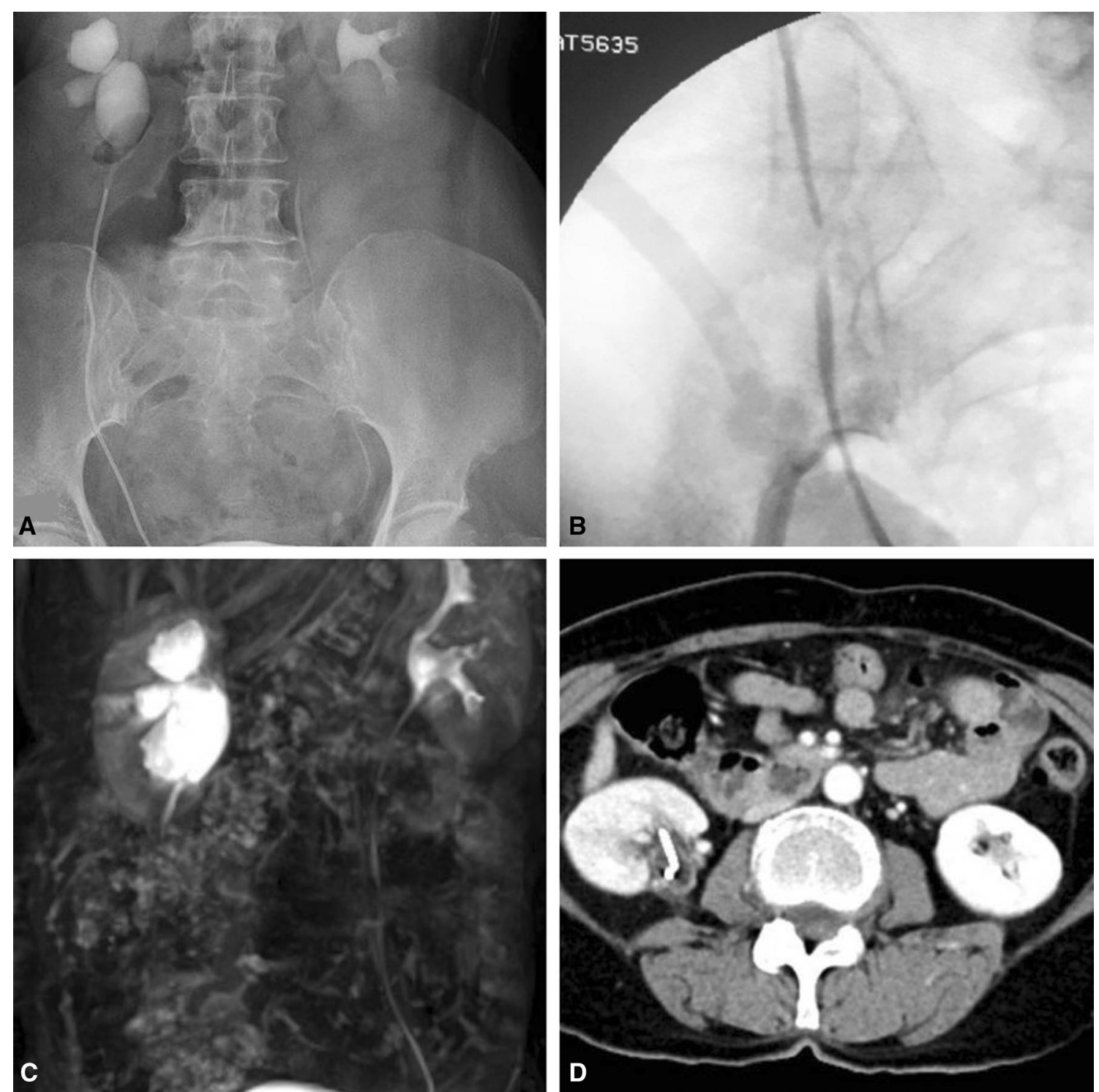

Fig. 2 Follow up images. a Postoperative 2nd-month IVU, b postoperative 3rd-month RGP, c postoperative 3rd-month MR urography, d postoperative 6th-month CT

the bladder was anastomosed to the ureter through ureteroneocystostomy. The omental flap was rotated. The surgery was ended after wrapping the whole ureter with the omental flap. Nephrostomy catheter was not inserted (Fig. 1).

\subsection{Follow-up and result}

The Foley catheter was removed on postoperative 15 day. Six months post-operation, D-J stent was removed and retrograde pyelography (RGP) was performed. Ureter was normal, and contrast entrance to renal pelvis was adequate. There was a small annular stricture in the distal ureter which required D-J stent re-insertion (Additional file 1 Video-1). The patient was followed up through ultrasonography, CT urography and scintigraphy. In addition to the presence of average hydronephrosis, split right kidney function was $21 \%$ on postoperative first-year scintigraphy. The patient was followed up with D-J stent for postoperative 1 year. Hydronephrosis progression and clinical pain were not noticed during the second-year followups without D-J (Fig. 2). 

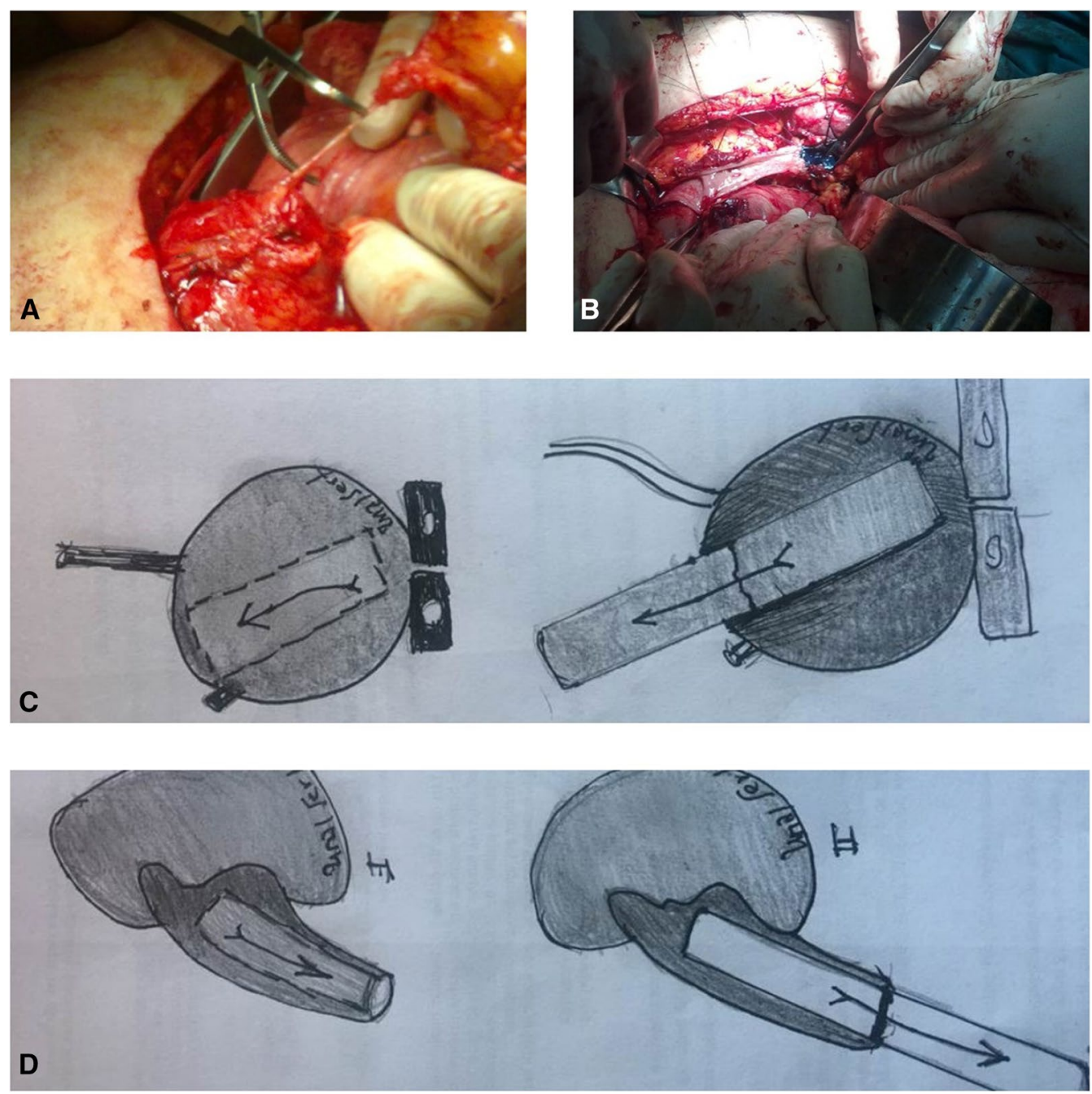

Fig. 3 Boari flap technique. a Avulsiye ureter, b boari flap \& renal pelvis flap anastomosis, c boari flap drawing, d renal pelvis flap drawing

\section{Case-2}

\subsection{Medical history}

A 59-year-old man had ureteroscopy in another hospital for an upper ureteral stone with a diameter of $10 \mathrm{~mm}$. The urologist stated that a 9F ureterorenoscope was used, and due to lack of experience, the patient was referred to our clinic with avulsion of the right ureter. Based on the CT urography taken in our hospital and the surgical story from the surgeon, total ureteral avulsion was detected in the patient and urgent surgery was planned.

\subsection{Surgical technique}

Modified paramedian incision was performed for repair. The retroperitoneum was entered, and the ureter was found and dissected. Anastomosis was performed between the avulsed ureter and UP junction. Double J stent was inserted, and bladder ureteroneocystostomy
(UNC) was performed. A nephrostomy catheter was inserted after wrapping the ureter with omental flap.

\subsection{Follow-up and result}

The patient was discharged on day 5 since no problems were noticed in the postoperative follow-ups. The Foley catheter was removed on postoperative day 10 while the nephrostomy catheter was removed in the postoperative first month. RGP was taken in the third month follow-up. No stricture was observed in UP and UV junctions, but there were crusty clots inside the ureter. These clots were washed, and D-J stent was re-inserted. RGP was repeated in postoperative sixth month. The ureter was in better condition, and there were no crusts and $7 f$ D-J stent was re-inserted. In patient followups, LUTS was detected after Foley catheter removal. It was learned from the patient that this condition was 
present before surgery. During examinations, prostate size was observed to be approximately $40 \mathrm{cc}$ on urinary USG. IPSS was 10, maximum flow was $10 \mathrm{ml} / \mathrm{s}$, mean flow rate was $5 \mathrm{ml} / \mathrm{s}$, and postvoid residue amount was $80 \mathrm{cc}$ on uroflowmetry. Alfuzosin was started and was combined with dutasteride due to the lack of significant recovery in the symptoms. TUR-P operation was performed at postoperative 1 year as there was no recovery in patient symptoms. The ureter was intact on URS and RGP of the patient performed before Tur-P.

Ultrasonography, abdominal CT and scintigraphy were used for patient follow-up. Although the previous condition of right kidney functions was unknown, $33 \%$ right kidney split function and mild hydronephrosis were detected based on postoperative scintigraphy. The patient is still being followed up in our follow-up program.

\section{Case-3}

\subsection{Medical history}

A 53-year-old man had ureteroscopy in another hospital for mid-ureteral stone with a diameter of $8 \mathrm{~mm}$. The urologist stated that $11 \mathrm{~F}$ ureterorenoscope and pneumatic lithotriptor were used, and due to lack of experience, the patient was referred to our clinic since he had avulsion of the left ureter. Based on the CT urography taken in our hospital and detailed medical history from the surgeon, total ureteral avulsion was detected in the patient and urgent surgery was planned.

\subsection{Surgical technique}

Modified paramedian incision was made for repair. Retroperitoneum was entered, and the ureter was found and dissected. Based on surgical observation, the kidney had wide extra renal pelvis and $y-v$ flap was rotated on the renal pelvis. Then, a boari flap was rotated on the bladder and ureter anastomosis was performed and D-J stent was inserted. A nephrostomy catheter was not inserted (Fig. 3).

\subsection{Follow-up}

Foley catheter was removed on postoperative day 15 . Anticholinergic treatment was given for bladder capacity. Retrograde pyelography (RGP) was performed after D-J stent removal 3 months postoperative. Although an average degree of hydronephrosis was observed, no extravasation was present. The D-J stent was re-inserted, and the patient was followed up with ultrasonography and abdominal CT. There was mild thinning in renal parenchyma, and moderate hydronephrosis continued. Based on scintigraphy, normal renal excretion and a mild decrease in left kidney functions were observed. D-J stent was changed in postoperative sixth month, and no stricture was observed in RGP. The D-J stent was removed 12 months postoperative.

Split function of the left kidney was $28 \%$ on scintigraphy taken in postoperative first year. The patient is still being followed up in our program. Data for the patients are provided in Table 1.

\section{Discussion}

The quick advancement of technology, improvement in instruments, new energy sources, better optic quality of devices and increased experience with ureteral calculi, ureter and kidney-related conditions in the last decades caused high success in endoscopic operations, and thus, ureteroscopy has become the first choice for ureteral calculi and ureter-related operations [8-10]. On the other hand, ureteroscopic operations may cause many complications such as residual calculi, mucosal injury, perforation, bleeding and edema [11]. Ureteral avulsion is a rare but extremely severe complication with a reported

\section{Table 1 Patients and operation characteristics}

\begin{tabular}{|c|c|c|c|}
\hline Case & I & II & III \\
\hline Age & 66 & 59 & 53 \\
\hline Sex & Female & Male & Male \\
\hline Diagnosis before URS & Hematuria + mid-ureter stone & Upper ureteral stone & Middle ureteral stone \\
\hline Side & Right & Right & Left \\
\hline Size of ureteroscope & $9 \mathrm{Fr}$ & $9 \mathrm{Fr}$ & $11 \mathrm{Fr}$ \\
\hline Operation & $\begin{array}{l}\text { Ureteroeplvic anastomosis + ureteroneocyc- } \\
\text { tostomy + omental wrapped }\end{array}$ & $\begin{array}{l}\text { Ureteroeplvic anastomosis + ureteroneocyc- } \\
\text { tostomy + omental wrapped }\end{array}$ & Y-v flap + Boari flap \\
\hline Intervention time & Immediately & Immediately & Immediately \\
\hline Follow-up & Moderate hydronephrosis + atrophy & Mild hydronephrosis & Mild hydronephrosis \\
\hline $\begin{array}{l}\text { Scintigraphy (split function of } \\
\text { effected kidney) }\end{array}$ & $21 \%$ & $33 \%$ & $28 \%$ \\
\hline
\end{tabular}


percentage of 0-3.75 [12]. The first avulsion case was reported by Hart and Hodge after manipulation with Dormia basket. Risk factors for avulsion are previous manipulations and high calculi load. Complication risk also increases with proximal ureteral calculi after extracorporeal shock wave lithotripsy (ESWL) treatment [13]. In addition to all these factors, basket use, inexperience, thick ( $>9$ F) ureterorenoscope use and ureteral inflammation can also be stated as risk factors [14]. These conditions were stated in studies in the literature. In our cases, ureteral inflammation and $>9 \mathrm{~F}$ ureterorenoscope use, especially, were prominent.

Predisposing factors are controversial for ureteral avulsion. But observance of intense inflammation in the ureter during ureteroscopy is among the important factors [15]. The presence of inflammatory tissue in proximal or distal ureter or the region where the stone was impacted forms a potential for ureteral wall rupture [16]. Added to this factor, the delicate tensile strength of the region was stated as $457.52-3374 \mathrm{~N} \mathrm{~cm}^{-2}$ in circumferential direction and as $902.43-122.08 \mathrm{~N} \mathrm{~cm}^{-2}$ in the longitudinal direction [16]. In our cases, the ureter, especially its distal end, was observed to be inflamed.

Treatment and management of ureteral avulsion are quite challenging. Traditionally, ureteral avulsion is surgically treated and the reconstructive technique changes according to the length and location of ureter. Ureteroneocyctostomy is the general treatment option for distal ureteral injuries. While psoas hitch or boari flap procedures can be preferred for central ureteral injuries, end-to-end anastomoses using D-J stent can be a treatment option for proximal ureteral injuries. Finally, ileal interposition or renal autotransplantation is generally required in large ureteral injuries with a significant amount of tissue loss [17]. If avulsion is noticed in the postoperative period, percutaneous nephrostomy can be located and definitive treatment can be given when the patient is stable [18]. Ureteral avulsion treatment and management are challenging and controversial. In the literature, nephrectomy was also reported as an option for extended ureteral injuries [19], while there are also limited options such as ileal interposition or renal autotransplantation and appendix interposition [20, 21]. But the number of publications on the approach to the ureter totally avulsed at both ends is still limited in the literature [22].

The management of major ureteral injury is always a challenge [23]. It depends on the location and extent of the injury. Renal autotransplantation is considered a suitable option for ureteral injuries, especially in cases with major ureteral length loss. Delayed recognition of injury requires treatment with extended procedures as well as high experience of the urologists. Repair of long-term defect of the ureter, especially of the proximal ureter, is a particularly difficult surgical challenge. No strict recommendations are available about the treatment of long ureteral lesions [24]. Early intervention was preferred in our cases. Transplantation was not considered since surgical experience is important.

Ordon et al. reported three cases they named as "the scabbard avulsion." Nephrostomy was inserted postoperatively, and nephrectomy was performed in all patients [19]. Ge et al. presented four ureteral avulsion cases, two of which were total ureteral avulsions. One of the cases was treated with autotransplantation, and no problem was noticed in the follow-up. In the other case, pyeloureterostomy was performed and extended omentum was wrapped, but nephrectomy was performed after a 25-month follow-up [5]. Thai et al. reported a series of six ureteral avulsion cases. Five of them had avulsion in UPJ and UVJ. The authors reported that they refused to perform nephrectomy in one patient and performed boari flap in two patients, with ileal interposition and ureteral reimplantation in one patient each [25]. Unsal et al. reported four ureteral avulsion cases and performed boari flap in one half and ureteral reimplantation procedures in the other half of these cases. During the followup for ureteral reimplantation patients, one was normal while one had hydronephrosis [3]. Sevinç et al. reported three total ureteral avulsion cases treated with reimplantation and ileal interposition [22]. Grzegółkowski et al. [26] reported that extended length flap from the bladder was used instead of the entire ureter with modified boari flap technique in a total ureteral avulsion case and watertight, tension free anastomosis was performed, and they also reported extended boari flap modification as a possible alternative reconstructive treatment for total ureteral losses.

We also had three "total ureteral avulsion in both ends" cases and treated two of these with ureterocaliostomy + unc + omental flap wrapping and performed boari flap + renal pelvis $\mathrm{y}-\mathrm{v}$ advancement on the other (Table 1). In our series, we observed that ureteral feeding was provided by wrapping the omentum around total avulsified ureter and we believe that the patient can perform normal ureteral functions and recover more easily as the intestinal system was not intervened. As one of our patients had an extra renal pelvis, we performed $y-v$ advancement from the renal pelvis and a new ureter was formed by rotating boari flap on the bladder. We believe boari flap is a good option, especially in cases in which psoas hitch is not able to reach or a new tube is formed. In this series, we think that it is important to perform ureteral reimplantation, especially in the early period, and to wrap the omental flap all around to feed the reimplanted ureter. 
The literature shows that it is suitable to completely wrap the ureter with omental flap in addition to pyeloureterostomy and ureterovesical anastomosis for all full-length ureteral avulsions and that omental flap feeds the avulsified ureter $[5,6]$. In line with previous studies and case presentations, we believe that wrapping the avulsified ureter with omental flap and performing pyeloureterostomy and ureterovesical anastomosis would be a good solution for complete ureteral avulsions [27]. We observed that the ureter was relieved on retrograde pyelographies in postoperative patient follow-ups and there was no extravasation in CT urography. Normal ureteral mucosa and good feeding were observed when diagnostic URS was performed for the patient who had total ureteral reimplantation + omental flap in postoperative sixth month. But a functional decrease and partial scar areas were detected on scintigraphy.

In the literature, 55\% [17] of 31 cases had avulsion of the right ureter and 45\% [14] had it in left ureter. [3-7, 12-27] Two of our cases had right ureter avulsion, and one patient had left ureter avulsion which was in line with the literature.

As a result, ureteral avulsion is hard to manage and is a nightmare for urologists. But it is important to stay calm and to refer the patient to a center experienced in this field or the injury should be cured in a suitable way together with another urologist, and in case of reimplantation, wrapping the re-implanted ureter with omentum to help its nutrition is an important trick. If ureteral avulsion is well managed, the patients can continue their normal lives without needing nephrectomy, although there is an acceptable loss of renal functions.

\section{Conclusion}

Ureteral avulsion management is an extremely difficult condition for both the surgeon and the patient. So, the most important thing is to prevent ureteral avulsion. Refraining from sharp and hard manipulations is a necessity in upper ureteral calculi and/or patients who previously had treatment like ESWL. Refraining from basket use as much as possible and maybe above all, inserting D-J stent in difficult conditions without forcing calculi and ureter or delaying to another session could be the best solution. But despite all precautions and experience, ureteral avulsion is a risk always faced by urologists. In cases of ureteral avulsion, the patient should be referred to experienced centers in this field and the condition should successfully be managed without panic. Ureteroplasty and omental flap treatment is an applicable method for complete ureteral avulsions with quite successful results.

\section{Supplementary information}

Supplementary information accompanies this paper at https://doi. org/10.1186/s12301-020-00047-4.

Additional file 1: Video-1. Postop 3rd-month control ureteroscopy video of ureteral re-implantation.

\section{Abbreviations}

URS: ureteroscopy; UPJ: ureteropelvic junction; D-J stent: double-J stent; RGP: retrograde pyelography; CT: computed tomography; UNC: ureteroneocystostomy; UVJ: uretero-vesical junction; TUR: transurethral resection; ESWL: extracorporeal shock wave lithotripsy.

\section{Acknowledgements}

Not applicable.

\section{Authors' contributions}

IUS designed the study and also performed supervision; AA prepared the manuscript and analyzed the data. All authors read and approved the final manuscript.

\section{Funding}

None of the authors involved in this study received financial support.

\section{Availability of data and materials}

The datasets used and/or analyzed during the current study are available from the corresponding author on reasonable request.

Compliance with ethical standards

\section{Competing interests}

The authors declare that they have no competing interests.

\section{Ethics approval and consent to participate}

Authors declared that the research was conducted according to the principles of the World Medical Association declaration of Helsinki "Ethical Principles for Medical Research Involving Human Subjects" (amended in October 2013). Ethics approval is not applicable. Consent for participation was obtained from all the patients.

\section{Consent for publication}

All patients included in this research gave written informed consent to publish the data contained within this study.

Received: 18 February 2020 Accepted: 24 June 2020

Published online: 19 October 2020

\section{References}

1. Pérez-Castro E, Martínez Piñeiro JA (1982) Ureteral and renal endoscopy. A new approach. Eur Urol 8:117-120

2. D'Addessi A, Bassi P (2011) Ureterorenoscopy: avoiding and managing the complications. Urol Int 87:251-259

3. Unsal A, Oguz U, Tuncel A et al (2013) How to manage total avulsion of the ureter from both ends: our experience and literature review. Int Urol Nephrol 45:1553-1560

4. Gupta V, Sadasukhi TC, Sharma KK et al (2005) Complete ureteral avulsion. Sci World J 28:125-127 
5. Ge C, Li Q, Wang L, Jin F, Li Y, Wan J et al (2011) Management of complete ureteral avulsion and literature review: a report on four cases. J Endourol 25:323-326

6. Gao P, Zhu J, Zhou Y, Shan Y (2013) Full-length ureteral avulsion caused by ureteroscopy: report of one case cured by pyeloureterostomy, greater omentum investment, and ureterovesical anastomosis. Urolithiasis 41:183-186

7. Chang SS, Koch MO (1996) The use of an extended spiral bladder flap for treatment of upper ureteral loss. J Urol 156:1981-1983

8. Wu CF, Shee JJ, Lin WY, Lin CL, Chen CS (2004) Comparison between extracorporeal shock wave lithotripsy and semirigid ureterorenoscope with holmium: YAG laser lithotripsy for treating large proximal ureteral stones. J Urol 172(5 Pt 1):1899-1902

9. Türk C, Knoll T, Petrik A et al (2019) Guidelines on urolithiasis. Available at http://www.uroweb.org/gls/pdf/21_UrolithiasisLRV4.pdf

10. Preminger GM, Tiselius HG, Assimos DG et al (2007) Management of ureteral calculi: EAU/AUA nephrolithiasis panel. http://www.auanet.org/ common/pdf/education/clinical-guidance/Ureteral-Calculi.pdf

11. Abdelrahim AF, Abdelmaguid A, Abuzeid H, Amin M, Mousa ES, Abdelrahim F (2008) Rigid ureteroscopy for ureteral stones: factors associated with intraoperative adverse events. J Endourol 22:277-280

12. Al-Awadi K, Kehinde EO, Al-Hunayan A, Al-Khayat A (2005) latrogenic ureteric injuries: incidence, aetiological factors and the effect of early management on subsequent outcome. Int Urol Nephrol 37:235-241

13. Fuganti PE, Pires S, Branco R, Porto J (2008) Predictive factors for intraoperative complications in semirigidureteroscopy:analysis of 1235 ballistic ureterolithotripsies. Urology 72:770-774

14. Martin X, Ndoye A, Konan PG et al (1998) Hazards of lumbar ureteroscopy: apropos of 4 cases of avulsion of the ureter. Prog Urol 8:358-362

15. de Andrade Junior GF, Barroso FMDS, Alves RADC, Marinho JG, Rodriguez JER, Mancini ALC (2020) Boari-Flap reconstruction due to a total ureteral avulsion in a patient with ipslateral ureterolithiasis and contralateral renal nodule. Urol Case Rep 30:101118. https://doi.org/10.1016/j. eucr.2020.101118

16. Shilo Y, Pichamuthu JE, Averch TD, Vorp DA (2014) First prize: evaluation of the tensile strength of the human ureter - preliminary results. J Endourol 28(12):1470-1473. https://doi.org/10.1089/end.2014.0226
17. Trinchieri A, Montanari E, Salvini P, Berardinelli L, Pisani E (2001) Renal autotransplantation for complete ureteral avulsion following lumbar disk surgery. J Urol 165:1210-1211

18. de la Rosette JJ, Skrekas T, Segura JW (2006) Handling and prevention of complications in stone basketing. Eur Urol 50:991-998

19. Ordon M, Schuler TD, Honey RJ (2011) Ureteral avulsion during contemporary ureteroscopic stone management: 'the scabbard avulsion'. J Endourol 25:1259-1262

20. Dagash H, Sen S, Chacko J et al (2008) The appendix as ureteral substitute: a report of 10 cases. J Pediatr Urol 4:14-19

21. Juma S, Nickel JC (1990) Appendix interposition of the ureter. J Urol 144:130-131

22. Sevinc C, Balaban M, Ozkaptan O, Yucetas U, Karadeniz T (2016) The management of total avulsion of the ureter from both ends: our experience and literature review. Arch Ital Urol Androl 88(2):97-100. https://doi. org/10.4081/aiua.2016.2.97

23. Benson MC, Ring KS, Olsson CA (1990) Ureteral reconstruction and bypass: experience with ileal interposition, the Boari flap-psoas hitch and renal autotransplantation. J Urol 143:20-23

24. Moulavasilis N, Katafigiotis I, Staios D, Nikolaidis C, Vernadakis S, Bokos J, Anastasiou I (2020) Renal autotransplantation: a final option to preserve the kidney after an iatrogenic ureteral injury. Arch Ital Urol Androl 91(4):263-264. https://doi.org/10.4081/aiua.2019.4.263

25. Taie K, Jasemi M, Khazaeli D et al (2012) Prevelance and management of complications of ureteroscopy: a seven-year experience with introduction of a new maneuver to prevent ureteral avulsion. Urol J 9:356-360

26. Grzegółkowski P, Lemiński A, Słojewski M (2017) Extended Boari-flap technique as a reconstruction method of total ureteric avulsion. Cent Eur J Urol 70(2):188-191. https://doi.org/10.5173/ceju.2017.927

27. Tang K, Sun F, Tian Y, Zhao Y (2016) Management of full-length complete ureteral avulsion. Int Braz J Urol 42(1):160-164. https://doi.org/10.1590/ S1677-5538.IBJU.2015.0372

\section{Publisher's Note}

Springer Nature remains neutral with regard to jurisdictional claims in published maps and institutional affiliations.

\section{Submit your manuscript to a SpringerOpen ${ }^{\circ}$ journal and benefit from:}

- Convenient online submission

- Rigorous peer review

- Open access: articles freely available online

- High visibility within the field

- Retaining the copyright to your article

Submit your next manuscript at $\boldsymbol{\nabla}$ springeropen.com 